\section{Mort neuronale dans les modèles expérimentaux de la maladie de Parkinson}

Nathalie Lambeng, Raphaël Hourez, Sakina Torch, Jean-Marc Verna, David Blum
> La caractérisation des mécanismes moléculaires impliqués dans la mort neuronale survenant au cours de la maladie de Parkinson idiopathique est indispensable pour l'élaboration de traitements capables d'arrêter l'évolution de cette maladie. Ces mécanismes ont essentiellement été décryptés grâce à deux modèles expérimentaux utilisant des neurotoxiques capables de reproduire les caractéristiques anatomopathologiques (dégénérescence des neurones dopaminergiques de la substance noire) et biochimiques (stress oxydatif et inhibition mitochondriale) de la maladie de Parkinson idiopathique. La mort cellulaire induite dans ces modèles est de type apoptotique. La phase finale de ce processus met en jeu l'activation de la caspase effectrice de type 3 . Les phases plus précoces semblent réglées par la protéine $p 53$ et les protéines pro- et anti-apoptotiques de la famille $\mathrm{Bcl}-2$ mais également modulées par le facteur transcriptionnel NFKB et les MAPkinases. Toutes ces étapes constituent autant de cibles thérapeutiques potentielles. <
La maladie de Parkinson idiopathique est une maladie neurodégénérative qui, étant donné le vieillissement de la population, représente un problème de santé publique d'une importance croissante. Son étiologie demeure à ce jour inconnue et sujette à controverses (facteurs toxiques endogènes ou exogènes, facteurs génétiques, vieillissement). Le diagnostic clinique repose sur l'identification de trois symptômes moteurs cardinaux : la rigidité, le tremblement et la bradykinésie (un trouble moteur se manifestant par des mouvements ralentis et la perte des mouvements fins).

Au point de vue anatomo-pathologique, la maladie de Parkinson se définit par : (1) une perte des neurones dopaminergiques de la substance noire; et (2) la pré-

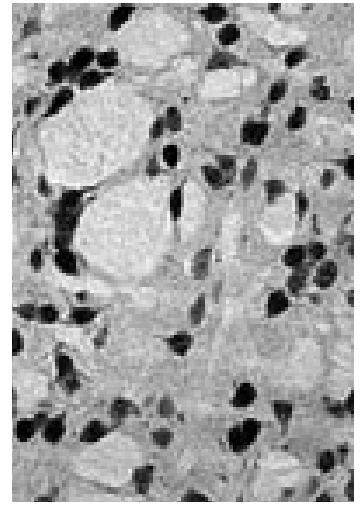

sence d'au moins un corps de Lewy, une inclusion intraneuronale éosinophile, au niveau de la substance noire et/ou du locus cœruleus [1, 2]. Malgré un nombre considérable d'études cliniques et expérimentales, les traitements de cette maladie, pharmacologiques (principalement la L-Dopa) ou chirurgicaux (par exemple, la stimulation du noyau sous-thalamique) n'agissent, jusqu'à preuve du contraire, que sur les symptômes et non pas sur l'évolution du processus dégénératif. Cela s'explique principalement par notre large méconnaissance des mécanismes intimes de la mort cellulaire impliqués dans la perte neuronale associée à la maladie de Parkinson et dont une meilleure compréhension devrait mener à la découverte de traitements neuroprotecteurs pharmacologiques ou géniques $(\rightarrow)$.

Les mécanismes de la mort des cellules nigrales sont étudiés principalement de deux manières. La première repose $\rightarrow) \mathrm{m} / \mathrm{s}$ $1997, n^{\circ} 4$, p. 617 
sur des études anatomo-pathologiques post-mortem de cerveaux de patients atteints de la maladie de Parkinson, la seconde sur des modèles expérimentaux reproduisant les caractéristiques lésionnelles et/ou biochimiques de la maladie humaine et qui permettent d'avoir une vision plus dynamique des phénomènes potentiellement impliqués. Différents modèles expérimentaux de la maladie de Parkinson ont été développés, tant in vitro qu'in vivo. II existe ainsi des modèles transgéniques visant à recréer les mécanismes dégénératifs observés dans les rares cas génétiques de la maladie. D’autres modèles tentent de reproduire l'action de toxiques exogènes supposés jouer un rôle étiologique dans la maladie de Parkinson (roténone, isoquinolines, manganèse). Toutefois, les modèles neurotoxiques les plus utilisés, en particulier dans de nombreuses études pré-cliniques, font appel à l'administration intracérébrale de 6-hydroxydopamine (6-OHDA) ou systémique de 1-méthyl-4-phényl-1,2,3,6-tétrahydropyridine (MPTP), deux neurotoxines dont l'implication en tant que facteur déclenchant n'est pourtant pas établie. Cet article a pour objectif de faire le point sur les mécanismes par lesquels la 6-OHDA et le MPTP provoquent la dégénérescence nigrale. Une première partie décrit globalement leur mode d'action, une seconde aborde les voies moléculaires (apoptotiques) impliquées dans leurs effets délétères.

\section{Bases biochimiques de la neurotoxicité induite par la 6-OHDA}

La 6-hydroxydopamine est un analogue hydroxylé de la dopamine isolé pour la première fois en 1959. Son premier effet décrit est une capacité d'induire une déplétion noradrénergique au niveau du système nerveux autonome cardiaque. Ses potentialités neurotoxiques ont ensuite été démontrées au niveau du système nerveux périphérique. Incapable de traverser la barrière hémato-encéphalique, la 6-OHDA ne peut induire de lésion nigrale chez le rat qu'après une injection stéréotaxique au niveau ventriculaire, parenchymateux directement dans la substance noire ou au niveau du faisceau médian antérieur reliant la substance noire au striatum - ou au niveau du striatum (lésion nigrale rétrograde). L'extension de la lésion dopaminergique ainsi engendrée peut être évaluée aisément par l'administration périphérique d'apomorphine ou d'amphétamine qui produit un comportement rotatoire transitoire chez les animaux ayant une lésion unilatérale, comportement pris pour index du degré de dégénérescence neuronale [3]. La 6-OHDA permet ainsi de produire des lésions nigrales apparentées à celles observées dans la maladie de Parkinson. Par ailleurs, différents travaux suggèrent une production physiologique de 6-OHDA par la substance noire, ce qui renforce l'intérêt pour ce modèle. La production intracellulaire de 6-OHDA impliquerait des mécanismes non enzymatiques faisant intervenir la dopamine, le peroxyde d'hydrogène et le fer libre [4], composés présents en forte concentration dans les neurones dopaminergiques (Figure 1). Cette production de 6-OHDA serait amplifiée : (1) par la mélanine, présente dans les cellules nigrales sensibles au processus dégénératif, et capable d'accroître les taux de fer libre en le libérant de la ferritine; (2) par la fraction de fer libéré de la ferritine par la neurotoxine elle-même [5]; et également (3) par les ions nitrites et le manganèse, eux-mêmes impliqués dans la dégénérescence nigrale. Chez le patient parkinsonien, la production nigrale de 6-OHDA serait accrue du fait d'une augmentation pathologique des taux de radicaux libres et de fer mais aussi par le traitement par la L-Dopa [6]. In vitro, l'utilisation de modèles expérimentaux faisant appel à diverses lignées cellulaires catécholaminergiques (lignées PC12, Sy5y, SKNSH, MN9D...) ou à des cultures primaires de neurones mésencéphaliques, a permis de démontrer que la toxicité de la 6-OHDA était principalement liée à sa capacité d'engendrer de fortes quantités de radicaux libres. D'autres études ont confirmé ces résultats in vivo $[2,3]$. Cela explique les effets protecteurs de nombreux anti-oxydants, ainsi que la plus grande résistance de souris transgéniques surexprimant la superoxyde dismutase ou la glutathion peroxydase $[7,8]$. L'origine de ces radicaux libres est multiple. D'une part, le peroxyde d'hydrogène, luimême producteur d'ions hydroxyles extrêmement réactifs, serait produit par la désamination de la 6-0HDA par la monoamine oxydase, l'une des principales enzymes de dégradation de la dopamine. Notons que ce mécanisme reste controversé. D'autre part, de fortes quantités de radicaux hydroxyles et de composés quinoniques seraient formées, principalement, par un mécanisme d'auto-oxydation non-enzymatique de la 6OHDA [9]. Enfin, in vivo, le processus dégénératif impliquerait une réaction microgliale capable de produire des radicaux libres dans les neurones dopaminergiques restants par l'intermédiaire d'une libération de TNF $\alpha$ (tumor necrosis factor $\alpha$ ). Dans tous les cas, la production de radicaux libres, en particulier de radicaux hydroxyles, est amplifiée par la présence de fer. Par conséquent, dans les neurones dopaminergiques, il existe un double mécanisme d'amplification de l'activité délétère de la 6-OHDA, le premier accroissant sa formation et le second aggravant ses effets oxydatifs. Indépendamment des phénomènes oxydatifs directs, différents travaux réalisés in vitro suggèrent que la 6-OHDA 
inhiberait l'activité mitochondriale. Ainsi, plusieurs études réalisées sur des préparations de mitochondries isolées démontrent que la 6-OHDA inhibe, d'une manière réversible et indépendante de la présence de fer, l'activité du complexe I de la chaîne respiratoire mitochondriale [10]. En outre, cette capacité d'inhiber le complexe I semble corrélée au comportement rotatoire induit chez des animaux ayant une lésion unilatérale. D'autres études démontrent que la neurotoxine est capable d'induire une chute du potentiel membranaire mitochondrial $(\Delta \psi \mathrm{m})$ et de provoquer le découplage de la phosphorylation oxydative d'une manière similaire au dinitrophénol. Néanmoins, de tels effets mitochondriaux directs sont conditionnés par la capacité de la 6-OHDA d'atteindre cet organite et donc d'entrer dans les cellules dopaminergiques. Concernant ce point, les données expérimentales sont très contradictoires [2]. L'analogie structurale de la 6-OHDA avec la dopamine, les effets neuroprotecteurs de certains inhibiteurs de la recapture de la dopamine ainsi que la corrélation existant entre la maturation des transporteurs dopaminergiques (TDA) et la toxicité de la 6-OHDA plaident en faveur d'un rôle prépondérant des transporteurs dopaminergiques dans les effets délétères de la neurotoxine. A contrario, d'autres études démontrent : (1) une toxicité de la 6-OHDA envers des cellules dépourvues de transporteurs dopaminergiques (neurones corticaux ou granulaires cérébelleux, lignées CHO, NIH3T3 ou (6) ; (2) I'inefficacité d'autres inhibiteurs de recapture de la dopamine ; (3) l'absence d'accumulation de la neurotoxine dans des cellules exprimant les transporteurs dopaminergiques; et (4) les effets protecteurs d'une activité catalase extracellulaire. Même si ces derniers résultats ont été obtenus essentiellement in vitro, conditions dans lesquelles la densité et l'activité des transporteurs dopaminergiques sont très différentes de ce qu'elles sont in vivo, il est difficile d'expliquer la prétendue spécificité de la toxicité de la 6-OHDA envers les neurones dopaminergiques uniquement par des mécanismes dépendant des transporteurs monoaminergiques. Cette question importante reste donc ouverte et les différentes hypothèses mécanistiques sont résumées dans la Figure 1 .

Finalement, les phénomènes oxydatifs et mitochondriaux induits par la 6-OHDA aboutissent à de nombreux événements délétères pour les neurones dopaminergiques, les précipitant vers la mort. L'intense stress oxydatif cellulaire provoque des altérations des systèmes de détoxification et du potentiel redox intracellulaire [11], la dépolymérisation de la tubuline, une forte diminution de la capture du glucose, mais surtout la peroxydation de macromolécules cellulaires parmi lesquelles les lipides et les acides nucléiques. Ainsi, in vivo, un traitement par la
6-OHDA provoque un accroissement des taux de malonyl-dialdéhyde [11] et une diminution des taux de phospholipides ainsi que des lésions de I'ADN mises en évidence par la formation de 8-hydroxy-2'-désoxyguanosine, de 5-hydroxycytosine et de 2,6-diamino,4hydroxy-5-formamidopyrimidine [12]. Les altérations mitochondriales, elles-mêmes productrices de radicaux superoxydes, induisent une diminution importante des taux d'ATP intracellulaire. Cela a pour principales conséquences, d'une part, d'altérer le stockage vésiculaire de la dopamine, ce qui provoque une augmentation cytosolique du neurotransmetteur et, d'autre part, de perturber la polarisation membranaire, ce qui induit une excitotoxicité indirecte accompagnée d'un dérèglement délétère de l'homéostasie calcique [13].

\section{Bases biochimiques de la neurotoxicité induite par le MPTP}

Le MPTP est une neurotoxine synthétique capable d'induire une dégénérescence nigrale dans plusieurs espèces animales. Sa découverte remonte au début des années 1980, à la suite de l'apparition de syndromes parkinsoniens irréversibles chez de jeunes toxicomanes américains. Ils avaient ingéré un dérivé synthétique du fentanyl, contenant environ $3 \%$ de MPTP [14]. L'analyse post-mortem des cerveaux de ces patients confirma la lésion de la substance noire, révélant ainsi I'intérêt expérimental du MPTP. II convient de noter que, contrairement à la 6-OHDA, la production de cette neurotoxine n'est pas endogène même si certaines hypothèses étiologiques suggèrent l'implication de composés chimiquement ou fonctionnellement proches $\mathrm{du}$ MPTP (isoquinolines, roténone) dans le déclenchement de la maladie de Parkinson humaine.

Que ce soit chez l'homme, le primate non humain ou la souris, le MPTP induit la perte des neurones dopaminergiques de la substance noire, ainsi qu'une diminution massive des quantités de dopamine dans le système nigro-striatal [3]. Chez les primates humains et non humains, en fonction du régime d'administration, le MPTP peut produire un syndrome parkinsonien qui reproduit presque toutes les caractéristiques de la maladie de Parkinson : tremblement, rigidité, bradykinésie et instabilité posturale (Tableau I) [15]. En revanche, le tremblement de repos n'est observé que chez le singe vert [15]. Chez le primate non humain, la topographie des lésions nigrales, après traitement par le MPTP, est similaire à celle observée chez le patient parkinsonien avec une atteinte préférentielle de la partie latérale de la substance noire [16]. D'autres animaux sont également sensibles au MPTP, en particulier la souris qui, bien que ne 
développant pas de symptomatologie caractéristique, est, de loin, la plus utilisée expérimentalement [3, 15]. Néanmoins, pour des doses comparables à celles utilisées chez la souris, l'administration de MPTP chez le rat ne produit pas de dégénérescence dopaminergique, suggérant que cet animal serait relativement insensible à cette neurotoxine, du moins lorsqu'elle est injectée par voie systémique.

Après injection périphérique, le MPTP traverse la barrière hémato-encéphalique puis est converti en son métabolite actif et toxique, le $\mathrm{MPP}^{+}$(1-méthyl-4-phénylpyridinium), par la monoamine oxydase $B$ présente dans les cellules gliales (Figure 1). Une fois relargué par ces dernières, le MPP+ est spécifiquement capté par les neurones dopaminergiques grâce aux transporteurs dopaminergiques. L'accumulation intracellulaire du $\mathrm{MPP}^{+}$est favorisée par la neuromélanine qui, en s'y complexant, retarde sa libération dans le cytoplasme [17], et par les transporteurs vésiculaires des monoamines (VMAT2) qui, en le confinant dans les vésicules synaptiques, réduisent sa concentration cytosolique et par conséquent ses effets délétères [18]. Le MPP ${ }^{+}$cytosolique est enfin transporté de manière active vers les mitochondries où il bloque l'activité du complexe I de la chaîne respiratoire, ce qui provoque la mort cellulaire. Comme pour la 6OHDA, l'inhibition du complexe I de la chaîne mitochondriale par le $\mathrm{MPP}^{+}$conduit à une diminution de la quantité d'ATP disponible, à une diminution du potentiel transmembranaire mitochon- drial, à une perturbation de l'homéostasie calcique et à la formation de radicaux libres $[2,3]$. Toutefois, même si l'inhibition mitochondriale peut à elle seule expliquer la toxicité du $\mathrm{MPP}^{+}$, certaines observations suggèrent un mécanisme d'action plus complexe, qui mettrait en jeu la formation de radicaux libres indépendamment de la mitochondrie. Les résultats obtenus sur des cellules dépour-

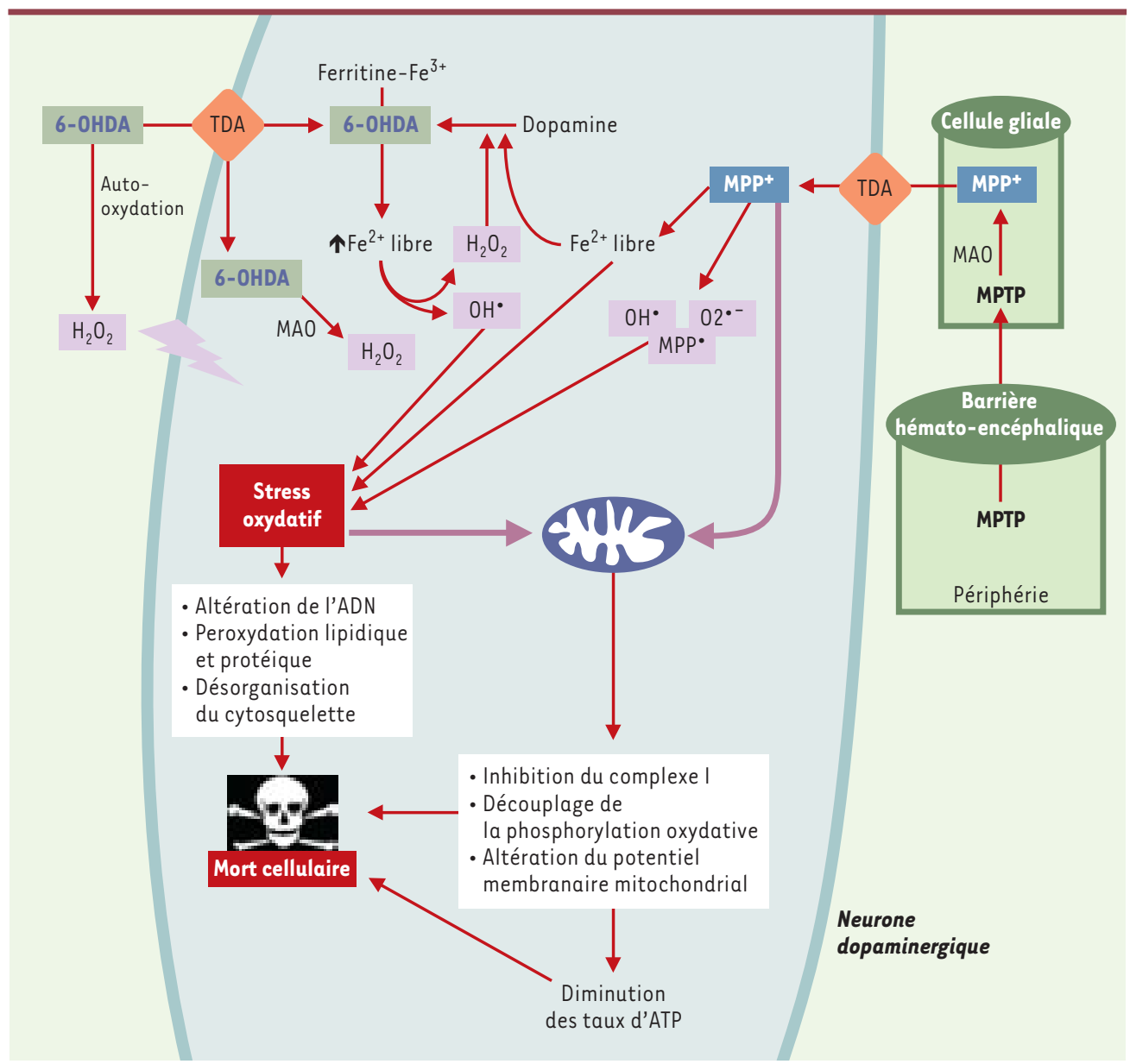

Figure 1. Mécanismes impliqués dans la toxicité de la 6-OHDA et du MPTP. L'accumulation de 6-OHDA dans les neurones dopaminergiques, à la suite d'une injection intracérébrale ou d'une synthèse endogène mettant en jeu la dopamine, la mélanine et le fer, conduit à : (1) des modifications mitochondriales (inhibition du complexe I de la chaîne respiratoire mitochondriale, découplage de la chaîne de phosphorylation oxydative et diminution du potentiel membranaire mitochondrial $[\Delta \psi \mathrm{m}]$ ), qui concourent à la diminution des taux d'ATP ; (2) la formation d'espèces radicalaires très réactives qui endommagent les macromolécules et participent à la désorganisation de la structure cellulaire. Ces événements conjugués conduisent à la mort des neurones. Notons qu'une oxydation de la 6-OHDA peut également avoir lieu à l'extérieur de la cellule, produisant alors du peroxyde d'hydrogène qui diffuse dans la cellule et concourt à l'accroissement des taux de radicaux libres intracellulaires. Le MPTP est, quant à lui, transformé en son métabolite actif, le $\mathrm{MPP}^{+}$, par la monoamine oxydase de type B présente dans les cellules gliales. Relargué par ces dernières, il est alors capté par les neurones dopaminergiques où il va, comme la 6-OHDA, inhiber le complexe I de la chaîne respiratoire mitochondriale, augmenter la synthèse de radicaux libres et provoquer la mort cellulaire. La diminution des taux d'ATP, à la suite du dysfonctionnement mitochondrial, est également responsable d'une augmentation de la concentration de calcium intracellulaire, liée à un phénomène d'excitotoxicité indirecte, qui accélère le processus de dégénérescence. 
vues d'une chaîne respiratoire fonctionnelle, mais qui sont toujours sensibles au $\mathrm{MPP}^{+}$, confirment cette possibilité [19]. Quoi qu'il en soit, la neurotoxicité du MPTP semble étroitement corrélée à la présence de radicaux libres dans la cellule. Le MPTP est à la fois capable de provoquer leur synthèse et de diminuer le contenu neuronal en molécules aidant à les éliminer comme le gluthation et la métallothionine. Des souris transgéniques surexprimant la superoxyde dismutase, une enzyme de détoxification des radicaux libres, sont moins sensibles à la toxicité du MPTP, et, inversement, l'effet du MPTP est potentialisé chez des souris déficientes pour cette protéine ou pour la glutathion peroxydase [20, 21]. Ici encore, le fer amplifie la synthèse de radicaux libres via le MPTP par des réactions non enzymatiques de type Fenton. Ce phénomène serait accru par la neurotoxine puisqu'elle est elle-même capable d'induire l'expression nigrale des récepteurs de la transferrine et des transporteurs de la lactoferrine. De plus, le MPP' peut produire directement des radicaux MPP ${ }^{\bullet}$ par une interaction avec la xanthine oxydase et des radicaux superoxydes par auto-oxydation du MPDP ${ }^{+}$, un métabolite du MPTP. D'autres observations suggèrent qu'une excitotoxicité indirecte, favorisée par le dysfonctionnement énergétique neuronal, conduirait à l'activation de la NOsynthase (monoxyde d'azote synthase) et à la formation de peroxynitrites [22]. Enfin, une activation microgliale productrice d'inflammation contribuerait à la synthèse de radicaux libres induite par le MPTP (Figure 1).

Il est important de noter que les études biochimiques et histo-pathologiques consacrées aux mécanismes toxiques de la 6-OHDA et du MPTP ont eu principale-

\begin{tabular}{|c|c|c|c|c|c|}
\hline & & MP & MPTP (singe) & MPTP (souris) & 6-OHDA (rat) \\
\hline \multirow{5}{*}{ 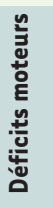 } & Akinésie & + & + & - & - \\
\hline & Rigidité & + & + & - & - \\
\hline & Tremblement & + & + & - & - \\
\hline & Tremblement de repos & + & $+a$ & - & - \\
\hline & Instabilité posturale & + & + & - & - \\
\hline \multirow{5}{*}{$\begin{array}{l}\frac{0}{00} \\
\frac{0}{0} \\
+\frac{1}{0} \\
0\end{array}$} & Lésion nigrale & + & + & + & + \\
\hline & Lésion ATV & + & + & + & - \\
\hline & Lésion LC & + & $\pm^{\mathrm{b}}$ & + & - \\
\hline & Corps de Lewy & + & $-c$ & - & - \\
\hline & Activation microgliale & + & + & + & + \\
\hline \multirow{5}{*}{ 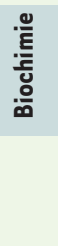 } & Diminution DA striatale & + & + & + & + \\
\hline & Stress oxydatif & + & + & + & + \\
\hline & Inhibition mitochondriale & + & + & + & + \\
\hline & Réponse à la L-Dopa & + & + & + & + \\
\hline & Durée de la dégénérescence & $\begin{array}{c}\text { Plusieurs années } \\
\text { à décennie }\end{array}$ & $\begin{array}{l}\text { Quelques } \\
\text { semaines }\end{array}$ & $\begin{array}{l}\text { Quelques } \\
\text { jours }\end{array}$ & $\begin{array}{l}\text { Quelques } \\
\text { jours }\end{array}$ \\
\hline
\end{tabular}

a. Chez le singe vert uniquement ; b. Selon l'espèce et le mode d'administration; c. Observés dans de très rares cas; ATV : aire tegmentale ventrale ; LC : locus cœruleus ; DA : dopamine ; MP : maladie de Parkinson ; MPTP : 1-méthyl-4-phényl 1,2,3,6-tétrahydropyridine ; 6-OHDA : 6-hydroxydopamine.

Tableau I. Similitudes et dissemblances entre la maladie de Parkinson idiopathique et les modèles neurotoxiques. ment pour objectif d'établir leurs similitudes et leurs dissemblances par rapport à la pathologie humaine (Tableau 1). Ce n'est pas le cas pour l'étude des mécanismes cellulaires intimes. En effet, les altérations moléculaires qui aboutissent à la mort des neurones dopaminergiques sont très peu connues chez l'homme. Seules quelques données factuelles ont été décrites (voir plus loin) (Tableau II). Une description dynamique de ces phénomènes a nécessité l'utilisation de ces modèles qui ont permis, tant in vitro qu'in vivo, la mise en évidence de facteurs potentiellement importants pour la dégénérescence nigrale humaine (Tableau II). Les modèles permettent ainsi une approche prospective des phénomènes moléculaires.

\section{Apoptose induite par la 6-OHDA et le MPTP}

L'implication d'un processus de mort cellulaire active, de nature apoptotique, dans la dégénérescence neuronale au cours de la maladie de Parkinson provient essentiellement d'expérimentations conduites sur des modèles in vitro. Il a ainsi été mis en évidence que la mort cellulaire induite par la 6-OHDA et le MPTP/MPP ${ }^{+}$mettait en jeu les acteurs bien connus de la phase finale d'exécution du processus apoptotique, par ailleurs impliqués dans d'autres types de dégénérescence neuronale (Figure 2, Tableau II). Ainsi, la perturbation du potentiel membranaire mitochondrial $(\Delta \psi \mathrm{m})$ par ces deux neurotoxines $[23,24]$ resitue leur mécanisme d'action dans le schéma classique du processus apoptotique, où la mitochondrie joue un rôle central, tant au niveau du contrôle de l'équilibre survie/mort, qu'au niveau du type de mort cellulaire, à savoir apoptose/nécrose [25]. Cette chute du $\Delta \psi \mathrm{m}$ est suivie de la libération dans le cytosol du cytochrome c [26] et de l'activation de la caspase effectrice de type 3 [27, 28]. Le maintien du $\Delta \psi \mathrm{m}$ retarde l'effet toxique du MPTP et I'inhibition pharmacologique ou génique de l'activité caspase protège de la mort cellulaire induite par le MPTP ou la 6-OHDA [29]. Signes fonctionnels de l'activation des caspases effectrices, la dégradation de la PARP (poly[ADPribose] polymérase) [12, 26], une protéine cible de ces protéases, ou l'activation de la PITSLRE kinase 
pl10, régulatrice de la transcription, ont été mises en évidence après traitement par les deux neurotoxines. De façon surprenante, le MPTP semble également activer la caspase 8 [30], une protéase régulatrice plutôt associée à une voie de signalisation activée par les récepteurs de mort (récepteur du TNFo, récepteur CD95/Fas). Cette observation pourrait toutefois s'expliquer par une expression accrue de cytokines pro-inflammatoires [31], en particulier de TNF $\alpha$, par les cellules microgliales lors du processus dégénératif, ce qui aurait tendance à amplifier la neurodégénérescence.

Inactiver les caspases, par l'utilisation d'inhibiteurs exogènes spécifiques ou la transfection des cellules nigrales par des gènes codant pour des inhibiteurs endogènes tels que les protéines IAP (inhibitory apoptosis protein) (XIAP, NIAP...) [32], pourrait ainsi constituer une stratégie thérapeutique intéressante pour la maladie de Parkinson. Toutefois, bien que favorisant la survie, l'inhibition de ces protéases ne semble pas per- $(\rightarrow) \mathrm{m} / \mathrm{s}$

2000, $n^{\circ} 2$,

p. 261 et

2001, $n^{\circ} 12$, p. 1356 mettre de maintenir la fonctionnalité des neurones dopaminergiques : leurs corps cellulaires sont protégés mais leurs terminaisons nerveuses dégradées [33, 34]. Les phases tardives du processus neurodégénératif ne représentent donc pas une cible thérapeutique adéquate. II serait a priori plus intéressant d'intervenir sur les étapes moléculaires plus précoces dont la nature n'est pour l'instant pas élucidée.

Deux types de molécules régulatrices du processus apoptotique semblent toutefois liées à la toxicité du MPTP et de la 6-OHDA : le facteur de transcription $\mathrm{p} 53$ et les protéines de la famille $\mathrm{Bcl}-2$ (Bax, $\mathrm{Bcl}-2$, $\left.\mathrm{Bcl}-\mathrm{x}_{\mathrm{L}}\right)(\rightarrow)$. Le MPTP et la 6-OHDA provoquent, in vitro, une augmentation précoce et transitoire de l'expression de la protéine p53 [35, 36]. De plus, la modulation de son expression par la staurosporine, l'acide rétinoïque, les astrocytes, ou son inactivation génique modifient la sensibilité cellulaire à ces neurotoxines $[37,38]$. Le facteur p53 semble par ailleurs capable, dans certaines conditions, de régler la fonction apoptotique de la protéine Bax, dont l'expression est induite en présence de 6OHDA [35], en permettant sa translocation vers la membrane mitochon- driale. Bax provoque alors la libération du cytochrome $c$ dans le cytosol et l'activation de la caspase 3 [39]. Les protéines de la famille $\mathrm{Bcl}-2$ et $\mathrm{p} 53$ semblent donc régler la mort cellulaire induite par la 6-OHDA et le MPTP.

D'autres voies de signalisation mettant en jeu le facteur de transcription NF-KB ou les MAP kinases pourraient également intervenir dans la dégénérescence nigrale. Bien que le rôle de NF-KB dans la régulation de l'apoptose ne soit pas encore clairement défini, son activation, après traitement par le MPTP ou la 6-OHDA, pourrait représenter un mécanisme protecteur contre les effets délétères de ces neurotoxines [40, 41]. De manière intéressante, $N F-\kappa B$ semble avoir pour cibles certains gènes de la famille $\mathrm{Bcl}-2$, comme $b c l-x_{L}$ et $b f l-$ $1 / A 1$ dont il augmente l'expression, de même que d'autres facteurs anti-apoptotiques comme les protéines IAP [42]. Parmi les MAP-kinases, l'activation des JNK (c-jun $N$-terminal kinases) par le MPTP ou la 6OHDA conduit à l'apoptose [43, 44]. D'autres MAPkinases, les protéines ERK (extra-cellular regulated kinases) pourraient être impliquées dans la toxicité de la 6-OHDA [45] mais, étant donné le rôle plutôt protecteur qui était attribué à ces kinases jusqu'à présent, ces résultats restent à confirmer.

Certains de ces résultats établis in vitro sur différentes lignées cellulaires catécholaminergiques ont pu être rapprochés de données obtenues in vivo dans les modèles neurotoxiques expérimentaux de la maladie de Parkinson, soulignant alors leur pertinence dans l'étude des mécanismes conduisant à la dégénérescence

\begin{tabular}{|c|c|c|c|c|c|c|}
\hline & & \multicolumn{2}{|c|}{$\begin{array}{l}\text { Modèles } \\
\text { in vitro }\end{array}$} & \multicolumn{2}{|c|}{$\begin{array}{l}\text { Modèles } \\
\text { in vivo }\end{array}$} & \multirow[t]{2}{*}{$\begin{array}{c}\text { Patients } \\
\text { parkinsoniens }\end{array}$} \\
\hline & & 6-OHDA & MPP $^{+}$ & 6-OHDA & MPTP & \\
\hline \multirow{5}{*}{ 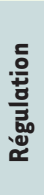 } & $\mathrm{Bcl}-2$ & Protège & Protège & Protège & Protège & ND \\
\hline & Bax & + & ND & ND & $+/$ Ko protecteur & ND \\
\hline & p53 & + & + & ND & Ko protecteur & + (striatum) \\
\hline & $N F-K B$ & + & + & ND & $=$ & + \\
\hline & JNK & + & + & ND & + & ND \\
\hline \multirow{2}{*}{ 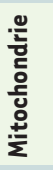 } & Chute $\Delta \psi \mathrm{m}$ & + & + & \multicolumn{3}{|c|}{....... Difficile à évaluer in vivo....... } \\
\hline & Cytochrome c & + & + & ND & + & ND \\
\hline \multirow[t]{2}{*}{ 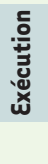 } & Activation caspase 3 & + & + & + & + & + \\
\hline & Morphologies apoptotiques & + & + & + & + & + \\
\hline
\end{tabular}

Tableau II. Modulation apoptotique dans les modèles de la maladie de Parkinson. 
nigrale. Ainsi, concernant la phase d'exécution de l'apoptose, l'activation de la caspase-3 a été mise en évidence dans les neurones dopaminergiques de souris traitées par le MPTP [46] ou de rats traités par la 6OHDA [47]. Parmi les protéines impliquées dans la phase de régulation du processus apoptotique, Bax est fortement exprimé dans la substance noire dopaminergique et l'invalidation de son gène protège des lésions induites par le MPTP [48]. Chez la souris traitée par le MPTP, la mort des neurones dopaminergiques coïncide avec l'induction de I'ARNm codant pour Bax et la diminution de l'expression de la protéine anti-apoptotique $\mathrm{Bcl}-2$. Cela concorde avec les effets neuroprotecteurs obtenus par la surexpression de $\mathrm{Bcl}-2[40,49]$ et, a contrario, avec une sensibilité accrue après invalidation de son gène [50]. Par ailleurs, les JNK sont également mises en jeu in vivo dans la dégénérescence nigrale après traitement par le MPTP [44, 51]. Dans ce contexte, si la phosphorylation des JNK, et donc leur activation, est inhibée, la perte neuronale est diminuée. Ces différentes kinases ainsi que le facteur NF-KB pourraient alors constituer des cibles privilégiées pour le développement de nouvelles stratégies thérapeutiques. Mais les rares données disponibles sur les mécanismes précis de la dégénérescence nigrale lors de la maladie de Parkinson, et notamment sur les étapes précoces, rendent indispensables de plus amples investigations. Il convient toutefois de préciser que certains des phénomènes moléculaires observés dans les modèles 6-OHDA et MPTP ont également été décrits chez le sujet parkinsonien, en particulier l'induction de Bax et l'activation de la caspase 3 [2]. L'activation du facteur NF- $K B$ a elle aussi été mise en évidence [52], mais aucune donnée concernant le rôle des JNK ou des ERK n'a été rapportée.

Certaines études plus précises, et bien que non dynamiques, pourraient toutefois devenir possibles grâce à de noucaspase 8 . Les flèches noires $(-\boldsymbol{l})$ représentent des phénomènes d'inhibition. 
velles méthodes moléculaires, en particulier l'analyse du transcriptome de cellules uniques prélevées par microdissection au laser.

Les données actuelles sont néanmoins insuffisantes pour établir les mécanismes moléculaires de la dégénérescence dopaminergique dans la substance noire dopaminergique des patients parkinsoniens, mais elles suggèrent l'existence de mécanismes de mort cellulaire communs à la maladie de Parkinson et aux modèles induits par des composés neurotoxiques comme la 6OHDA et le MPTP. Ces cascades moléculaires ne sont pourtant pas strictement spécifiques de la maladie de Parkinson puisque la contribution de $\mathrm{p} 53$, des protéines de la famille de Bcl-2, des caspases et du facteur NF$\kappa B$ a été suggérée dans d'autres maladies neurodégénératives comme la maladie d'Alzheimer.

\section{Pertinence et limites des modèles}

Finalement, même si leur mode d'action est sensiblement différent - la 6-OHDA étant plutôt un producteur radicalaire et le $\mathrm{MPP}^{+}$un inhibiteur mitochondrial - les effets de ces deux neurotoxines reproduisent la lésion histologique principale de la maladie de Parkinson mais également les deux phénomènes les mieux mis en évidence chez l'homme : un stress oxydatif co-dépendant des taux de fer libre et l'inhibition du complexe I mitochondrial. Au point de vue moléculaire, les données actuellement disponibles démontrent que leurs voies d'action sont assez similaires et correspondraient à ce qui est observable chez l'homme. Il ne faut toutefois pas perdre de vue qu'il ne s'agit que de modèles, par définition imparfaits (Tableau 1). Les études in vitro ne reproduisent à l'évidence pas les facteurs contextuels pouvant influer sur la dégénérescence neuronale. In vivo, au point de vue comportemental, l'administration de 6-OHDA chez le rat ou de MPTP chez la souris ne reproduit aucun des symptômes cardinaux de la maladie de Parkinson. Histologiquement, malgré de rares cas décrits chez le primate, et à la différence des modèles transgéniques surexprimant

$(\rightarrow) \mathrm{m} / \mathrm{s}$ 2000, n०8-9, p. 956 la protéine $\alpha$-synucléine $(\rightarrow)$, ces neurotoxines n'induisent pas la formation nigrale de corps de Lewy. De plus, les lésions extranigrales ainsi que la topographie lésionnelle, dans la mesure où l'anatomie est comparable, ne sont pas reproduites par la 6-OHDA chez le rat. Enfin, et surtout, ces modèles pêchent par leur inaptitude à reproduire l'aspect cinétique de la maladie humaine - décennies versus au mieux plusieurs semaines - et la possible contribution de phénomènes compensatoires à long terme dans le processus dégénératif.
Ces modèles constituent néanmoins un formidable tremplin expérimental pour l'étude des mécanismes fondamentaux sous-tendant la dégénérescence nigrale (apoptose). De manière importante, ils ont permis et permettent encore d'évaluer certaines options pré-cliniques et d'établir leur bien-fondé. De nombreux travaux sur les greffes de neurones mésencéphaliques fœtaux ont été réalisés dans ces modèles avant d'être envisagés chez l'homme. Certaines études utilisant les modèles, le singe MPTP en particulier, ont été déterminantes pour le développement des stimulations cérébrales profondes [53]. Actuellement, la pertinence d'approches neurotrophiques par thérapies cellulaire ou génique est testée activement dans les modèles neurotoxiques, tant in vitro qu'in vivo. Il faut donc admettre que, malgré leurs imperfections indéniables, les modèles expérimentaux neurotoxiques sont actuellement le seul recours fondamental permettant à terme de définir de nouvelles approches neuroprotectrices visant à stopper l'évolution de la maladie de Parkinson chez l'homme.

\section{REMERCIEMENTS}

Les auteurs remercient, pour leur soutien, l'Inserm, l'Université Joseph Fourier de Grenoble, la Fondation pour la Recherche Médicale, la Fondation Simone et Cino del Duca et le Fonds National pour la Recherche Scientifique (Belgique). Nous remercions également le Dr Marie-Christine Galas et le Pr JeanJacques Vanderhaeghen pour leurs commentaires avisés ainsi que Madeleine André pour la relecture attentive du manuscrit.

\section{SUMMARY}

Biochemical and molecular mechanisms of neuronal cell death in the experimental neurotoxic models of Parkinson's disease

A better knowledge of molecular pathways involved in the neuronal cell death occurring in Parkinson's disease is essential to achieve therapeutics able to block the degenerative process. These pathways have been partially elucidated using experimental models created by either 6-hydroxydopamine (6-OHDA) or 1-methyl-4phenyl-1,2,3,6-tetrahydropyridine (MPTP) which are able to mimick the histological (death of dopaminergic nigral neurons) and biochemical (oxidative stress and mitochondrial inhibition) hallmarks of Parkinson's disease. Cell death induced by these neurotoxins is thought to be apoptotic. At the very end-stage, this process depends on activated caspase-3. At earlier stages, in these models, cell death is regulated by $\mathrm{p} 53$ and $\mathrm{Bcl}-2$ family proteins but also by the transcriptional factor NF-KB and MAP-kinases. Modulating such regulatory processes may have potential therapeutic benefit for Parkinson's disease. $\Delta$ 


\section{RÉFÉRENCES}

1. Agid Y, Ruberg M, RaismanVozari R, Hirsch EC, JavoyAgid F. The biochemistry of Parkinson's disease. In: Stern GM, ed. Parkinson's disease. London : Chapman and Hall, 1990 : 99-125.

2. Blum D, Torch S, Lambeng $\mathrm{N}$, et al. Molecular pathways involved in the neurotoxicity of 6-OHDA, dopamine and MPTP : contribution to the apoptotic theory in Parkinson's disease. Prog Neurobiol 2001 ; 65 : 135-72.

3. Gerlach M, Riederer P. Animal models of Parkinson's disease : an empirical comparison with the phenomenology of the disease in man. J Neural Transm 1996 ; 103 : 987-1041.

4. Jellinger $K$, Linert $L$, Kienzl $\varepsilon$, Herlinger $\varepsilon$, Youdim MB. Chemical evidence for 6 hydroxydopamine to be an endogenous toxic factor in the pathogenesis of Parkinson's disease. J Neural Transm 1995 ; 46 (suppl) : 297-314.

5. Rocha ME, Ferreira AM, Bechara EJ. Roles of phosphate and an enoyl radical in ferritin iron mobilization by 5-aminolevulinic acid. Free Radic Biol Med 2000 ; 29 : 1272-9.

6. Andrew R, Watson DG, Best SA, Midgley JM, Wenlong $\mathrm{H}$, Petty RK. The determination of hydroxydopamines and other trace amines in the urine of parkinsonian patients and normal controls. Neurochem Res 1993 ; 18 : 1175-7.

7. Asanuma M, Hirata $H$, Cadet JL. Attenuation of 6hydroxydopamine-induced dopaminergic nigrostriatal lesions in superoxide dismutase transgenic mice. Neuroscience 1998 ; 85 : 907-17.

8. Bensadoun JC, Mirochnitchenko 0, Inouye
M, Aebischer P, Zurn AD. Attenuation of 6-OHDAinduced neurotoxicity in glutathione peroxidase transgenic mice. EurJ Neurosci 1998 ; 10 : 3231-6.

9. Graham DG, Tiffany SM, Bell WR Jr, Gutknecht WF. Autoxidation versus covalent binding of quinones as the mechanism of toxicity of dopamine, 6-hydroxydopamine, and related compounds toward C1300 neuroblastoma cells in vitro. Mol Pharmacol $1978 ; 14: 644-53$.

10. Glinka Yy, Youdim MB. Inhibition of mitochondrial complexes I and IV by 6hydroxydopamine. EurJ Pharmacol 1995 ; 292 : 329-32.

11. Kumar R, Agarwal ML, Seth PK. Free radical-generated neurotoxicity of 6-hydroxydopamine. J Neurochem 1995 ; 64 : 1703-7.

12. Bruchelt G, Schraufstatter IU, Niethammer D, Cochrane CG. Ascorbic acid enhances the effects of 6-hydroxydopamine and H2O2 on iron-dependent DNA strand breaks and related processes in the neuroblastoma cell line SK-N-SH. Cancer Res 1991; 51: 6066-72.

13. Brouillet $\varepsilon$, Peschanski $M$, Hantraye $P$. Du gène à la maladie : la mort neuronale dans la maladie de Huntington. Med Sci $2000 ; 16: 57-63$

14. Davis GC, Williams AC, Markey SP, et al. Chronic Parkinsonism secondary to intravenous injection of meperidine analogues. Psychiatr Res 1979 ; 1 : 249-54.

15. Przedborski $S$, JacksonLewis V, Naini AB, et al. The parkinsonian toxin 1 methyl-4-phenyl-1,2,3,6tetrahydropyridine (MPTP) : a technical review of its utilility and safety. J Neurochem 2001 ; 76 : 1265-74.
16. Varastet $M$, Riche $D$, Maziere M, Hantraye P. Chronic MPTP treatment reproduces in baboons the differential vulnerability of mesencephalic dopaminergic neurons observed in Parkinson's disease. Neuroscience 1994 ; 63 : 47-56.

17. D'Amato RJ, Alexander GM, Schwartzman RJ, Kitt CA, Price DL, Snyder SH. Evidence for neuromelanin involvement in MPTPinduced neurotoxicity. Nature 1987 ; 327 : 324-6.

18. Staal RG, Sonsalla PK. Inhibition of brain vesicular monoamine transporter (VMAT2) enhances 1methyl-4-phenylpyridinium neurotoxicity in vivo in rat striata. J Pharmacol Exp Ther 2000 ; 293 : 336-42.

19. Przedborski S, JacksonLewis V. Mechanisms of MPTP toxicity. Mov Disord 1998 ; 13 (suppl 1) : 35-8.

20. Klivenyi $P$, Andreassen $O A$, Ferrante RJ, et al. Mice deficient in cellula glutathione peroxidase show increased vulnerability to malonate, 3-nitropropionic acid, and 1-methyl-4-phenyl1,2,5,6-tetrahydropyridine. J Neurosci $2000 ; 20: 1-7$.

21. Zhang J, Graham DG, Montine TJ, Ho YS. Enhanced N-methyl-4phenyl-1,2,3,6tetrahydropyridine toxicity in mice deficient in $\mathrm{Cu} / \mathrm{Zn}$ superoxide dismutase or glutathione peroxidase. J Neuropathol Exp Neurol $2000 ; 59: 53-61$

22. Hantraye $P$, Brouillet $\varepsilon$, Ferrante $\mathrm{R}$, et al. Inhibition of neuronal nitric oxide synthase prevents MPTPinduced parkinsonism in baboons. Nat Med 1996 ; 2: 1017-21.

23. Lotharius J, Dugan LL, O'Malley KL. Distinct mechanisms underlie neurotoxin-mediated cell death in cultured dopaminergic neurons. J Neurosci 1999 ; 19 :
1284-93.

24. Cassarino DS, Parks JK, Parker WD Jr, Bennett JP Jr. The parkinsonian neurotoxin MPP+ opens the mitochondrial permeability transition pore and releases cytochrome $\mathrm{c}$ in isolated mitochondria via an oxidative mechanism. Biochim Biophys Acta 1999 ; 1453 : 49-62.

25. Yuan J, Yankner BA. Apoptosis in the nervous system. Nature 2000 ; 407 : 802-9.

26. Leist M, Volbracht C, Fava $\varepsilon$, Nicotera P. 1-Methyl-4phenylpyridinium induces autocrine excitotoxicity, protease activation, and neuronal apoptosis. Mol Pharmacol 1998 54 : 789-801.

27. Dodel RC, Du Y, Bales KR, Ling Z, Carvey PM, Paul SM. Caspase-3-like proteases and 6-hydroxydopamine induced neuronal cell death. Brain Res Mol Brain Res 1999 ; 64 : 141-8.

28. Du Y, Dodel RC, Bales KR, Jemmerson R, HamiltonByrd $\varepsilon$, Paul SM. Involvement of a caspase3 -like cysteine protease in 1-methyl-4phenylpyridinium-mediated apoptosis of cultured cerebellar granule neurons. J Neurochem 1997 ; 69 : 1382-8.

29. Ochu દE, Rothwell NJ, Waters CM. Caspases mediate 6hydroxydopamine-induced apoptosis but not necrosis in $\mathrm{PCl} 2$ cells. J Neurochem. $1998 ; 70: 2637-40$.

30. Hartmann A, Troadec JD, Hunot $S$, et al. Caspase- 8 is an effector in apoptotic death of dopaminergic neurons in Parkinson's disease, but pathway inhibition results in neuronal necrosis. J Neurosci 2001 ; 21 : 2247-55.

31. Nagatsu T, Mogi M, Ichinose $H$, Togari A. Changes in cytokines and neurotrophins 
in Parkinson's disease.

J Neural Transm 2000 ; 60

(suppl) : 277-90.

32. Crocker SJ, Wigle N, Liston

$P$, et al. NAIP protects the nigrostriatal dopamine pathway in an intrastriatal 6-OHDA rat model of Parkinson's disease. EurJ Neurosci 2001 ; 14 : 391-400.

33. Eberhardt 0 , Coelln RV, Kugler $\mathrm{S}$, et al. Protection by synergistic effects of adenovirus-mediated $\mathrm{X}$ chromosome- linked inhibitor of apoptosis and glial cell line-derived neurotrophic factor gene transfer in the 1-methyl-4phenyl-1,2,3,6tetrahydropyridine model of Parkinson's disease. J Neurosci $2000 ; 20$ : 9126-34.

34. von Coelln R, Kugler S, Bahr M, Weller M, Dichgans J, Schulz JB. Rescue from death but not from functional impairment: caspase inhibition protects dopaminergic cells against 6-hydroxydopamineinduced apoptosis but not against the loss of their terminals. J Neurochem $2001 ; 77: 263-73$.

35. Blum D, Wu Y, Nissou MF, Arnaud S, Alim LB, Verna JM. p53 and Bax activation in 6-hydroxydopamineinduced apoptosis in PC12 cells. Brain Res 1997 ; 751 : 139-42.

36. Kitamura Y, Kosaka T, Kakimura Jl, et al. Protective effects of the antiparkinsonian drugs talipexole and pramipexole against 1-methyl-4phenylpyridinium-induced apoptotic death in human neuroblastoma SH-Sy5Y cells. Mol Pharmacol 1998 ; 54 : 1046-54.

37. Trimmer PA, Smith TS, Jung $A B$, Bennett JP Jr. Dopamine neurons from transgenic mice with a knockout of the $p 53$ gene resist MPTP neurotoxicity. Neurodegeneration 1996 ; 5: 233-9.

38. Tieu K, Zuo DM, Yu PH.
Differential effects of staurosporine and retinoic acid on the vulnerability of the SH-Sy5y

neuroblastoma cells : involvement of bcl-2 and p53 proteins. J Neurosci Res 1999 ; 58 : 426-35.

39. Kroemer G, Reed JC. Mitochondrial control of cell death. Nat Med 2000 ; $6:$ 513-9.

40. Blum D, Torch S, Nissou MF, Verna JM. 6-

hydroxydopamine-induced nuclear factor-kappa B activation in $\mathrm{PCl} 2$ cells. Biochem Pharmacol 2001 ; $62:$ 473-81.

41. Cassarino DS, Halvorsen EM, Swerdlow RH, et al. Interaction among mitochondria, mitogenactivated protein kinases, and nuclear factor-kappaB in cellular models of Parkinson's disease. J Neurochem 2000 ; 74 : 1384-92.

42. Barkett M, Gilmore TD. Control of apoptosis by Rel/NF-kappaB transcription factors. Oncogene 1999 ; 18 : 6910-24.

43. Choi WS, Yoon Sy, Chang II, et al. Correlation between structure of $\mathrm{Bcl}-2$ and its inhibitory function on JNK and caspase activity in dopaminergic neuronal apoptosis. J Neurochem 2000 ; 74 : 1621-6.

44. Xia XG, Harding $T$, Weller $M$, Bieneman A, Uney JB, Schulz JB. Gene transfer of the JNK interacting protein-l protects dopaminergic neurons in the MPTP model of Parkinson's disease. Proc Natl Acad Sci USA 2001 ; 98 : 10433-8.

45. Kulich SM, Chu CT. Sustained extracellular signal-regulated kinase activation by $6-$ hydroxydopamine : implications for Parkinson's disease. J Neurochem $2001 ; 77$ : 1058-66.

46. Turmel $H$, Hartmann $A$, Parain K, et al. Caspase-3 activation in 1-methyl-4- phenyl-1,2,3,6-

tetrahydropyridine (MPTP)treated mice. Mov Disord 2001 ; 16 : 185-9.

47. Jeon BS, Kholodilov NG, Oo $\mathrm{TF}$, et al. Activation of caspase- 3 in developmental models of programmed cell death in neurons of the substantia nigra. J Neurochem 1999 ; $73: 322-33$.

48. Vila M, Jackson-Lewis V, Vukosavic S, et al. Bax ablation prevents dopaminergic neurodegeneration in the 1-methyl-4-phenyl1,2,3,6-tetrahydropyridine mouse model of Parkinson's disease. Proc Natl Acad Sci USA 2001 ; 98 : 2837-42.

49. Offen D, Beart PM, Cheung NS, et al. Transgenic mice expressing human $\mathrm{Bcl}-2$ in their neurons are resistant to 6-hydroxydopamine and 1-methyl-4-phenyl1,2,3,6-tetrahydropyridine neurotoxicity. Proc Natl Acad Sci USA 1998 ; 95 : 5789-94.

50. Hochman A, Sternin H, Gorodin $S$, et al. Enhanced oxidative stress and altered antioxidants in brains of $\mathrm{Bcl}-2$ - deficient mice. J Neurochem 1998 ; 71 : 741-8.

51. Saporito MS, Thomas BA, Scott RW. MPTP activates c-Jun NH(2)-terminal kinase (JNK) and its upstream regulatory kinase MKK4 in nigrostriatal neurons in vivo. J Neurochem 2000 ; 75 : 1200-8.

52. Hunot S, Brugg B, Ricard D, et al. Nuclear translocation of NF-kappaB is increased in dopaminergic neurons of patients with Parkinson disease. Proc Natl Acad Sci USA 1997 ; 94 : 7531-6.

53. Bergman $H$, Wichmann $T$, DeLong MR. Reversal of experimental parkinsonism by lesions of the subthalamic nucleus. Science 1990 ; 249 : 1436-8.

TIRÉS À PART

D. Blum et N. Lambeng 